\title{
H-function extension of the NBD in the light of experimental data
}

\author{
S. Hegyi \\ KFKI Research Institute for Particle and Nuclear Physics \\ of the Hungarian Academy of Sciences, \\ H-1525 Budapest 114, P.O. Box 49. Hungary \\ (October 10, 2018)
}

\begin{abstract}
The recently introduced $\mathrm{H}$-function extension of the Negative Binomial Distribution is investigated. The analytic form of $P_{n}$ is rederived by means of the Mellin transform. Applications of the HNBD are provided using experimental data for $P_{n}$ in $e^{+} e^{-}, e^{+} p$, inelastic $p p$ and non-diffractive $p \bar{p}$ reactions.
\end{abstract}

In multiparticle phenomenology we celebrate the 25th anniversary year of the invention of two ideas which turned out to be highly influential: the Koba-Nielsen-Olesen (KNO) scaling of the multiplicity distributions $P_{n}$ [1] and their Negative Binomial Distribution (NBD) type parametrization [2]. Both subjects received a great deal of attention during the past 25 years, see refs. [3-5] for review articles.

A popular way of introducing the NBD as a model of $P_{n}$ is the Poisson transform defined by

$$
P_{n}=\int_{0}^{\infty} \psi(z) \frac{z^{n}}{n !} e^{-z} d z .
$$

For the NBD the continuous probability density $\psi(z)$ is the gamma distribution providing the asymptotic KNO scaling form of $P_{n}$ [3]. At high energies the agreement between the NBD and observations is often not quite satisfactory therefore it is of interest to look for possible generalizations. We shall consider here an extension of the NBD that can be obtained via the scaling transformation $z \rightarrow z^{1 / \mu}$ of the gamma variate with scaling exponent $\mu>0$. The modified $\psi(z)$ can be written in the form

$$
\psi(z)=\frac{\mu}{\Gamma(k)} \lambda^{\mu k} z^{\mu k-1} \exp \left(-[\lambda z]^{\mu}\right)
$$

which is the generalized gamma distribution [6] with shape parameter $k>0$ and scale parameter $\lambda>0$. Its moments are given by

$$
\left\langle z^{q}\right\rangle=\int_{0}^{\infty} z^{q} \psi(z) d z=\frac{\Gamma(k+q / \mu)}{\Gamma(k)} \frac{1}{\lambda^{q}} .
$$

For $\mu=1$ the ordinary gamma distribution is recovered and the Poisson transform of Eq. (2) yields the NBD,

$$
P_{n}=\frac{1}{n !} \frac{\Gamma(k+n)}{\Gamma(k)}\left(\frac{\langle n\rangle}{k}\right)^{n}\left(1+\frac{\langle n\rangle}{k}\right)^{-k-n}
$$

where $\langle n\rangle$ is the average multiplicity. For $\mu \neq 1$ the basic properties of the Poisson transformed generalized gamma density have been determined in two previous Letters [7,8]. Since the analytic form of $P_{n}$ can be expressed in terms of the H-function of Fox [9] (see the Appendix for a summary) we will call the distribution as HNBD for short. The main goal of the present paper is the analysis of various multiplicity data which are known to be in disagreement with the $\mu=1$ special case.

In ref. [7] the analytic form of the HNBD was obtained utilizing the Laplace transform of the Fox function given by the equation pair (A.6-7). This is a straightforward method since $n ! P_{n}$ in Eq. (1) is the Laplace transform of the product $z^{n} \psi(z)$ which can be expressed in terms of $\mathrm{H}(x)$ for the generalized gamma distribution. To illustrate the simplicity of manipulating $\mathrm{H}$-functions, first we provide a slightly different approach to the analytic form of the newly developed probability law. The Laplace and Mellin transforms of a function $f(x), x \in(0, \infty)$, are defined by

$$
\mathcal{L}(f(x), r)=\int_{0}^{\infty} e^{-r x} f(x) d x
$$

and by

$$
\mathcal{M}(f(x), s)=\int_{0}^{\infty} x^{s-1} f(x) d x
$$


respectively. They are related to each other through

$$
\mathcal{M}\{\mathcal{L}(f(x), r), s\}=\Gamma(s) \mathcal{M}\{f(x), 1-s\}
$$

which can be justified by Fubini's theorem [10]. From the moments of $\psi(z)$ given by Eq. (3) one obtains

$$
\mathcal{M}\left\{z^{n} \psi(z), s\right\}=\frac{\Gamma(k+(s+n-1) / \mu)}{\Gamma(k)} \frac{1}{\lambda^{s+n-1}}
$$

for the Mellin transform of $z^{n} \psi(z)$ and the use of Eq. (7) yields

$$
\mathcal{M}\left\{\mathcal{L}\left(z^{n} \psi(z), r\right), s\right\}=\frac{1}{\lambda^{n} \Gamma(k)} \Gamma(s) \Gamma(k+n / \mu-s / \mu) \lambda^{s} .
$$

Comparison with the Mellin transform (A.8) of the Fox function shows that $\mathcal{L}\left(z^{n} \psi(z), r=1\right)$, providing $n ! P_{n}$, is expressible in terms of $\mathrm{H}(x)$ with the following parameters:

$$
\int_{0}^{\infty} e^{-z} z^{n} \psi(z) d z=\frac{1}{\lambda^{n} \Gamma(k)} \mathrm{H}_{1,1}^{1,1}\left[\frac{1}{\lambda} \mid \begin{array}{c}
(1-k-n / \mu, 1 / \mu) \\
(0,1)
\end{array}\right] \quad \text { for } \mu>1
$$

where the restriction on $\mu$ follows from the existence condition $\kappa>0$ of $\mathrm{H}(x)$ discussed in the Appendix. Thus, using identity (A.4), one arrives at

$$
P_{n}=\frac{1}{n ! \Gamma(k)} \mathrm{H}_{1,1}^{1,1}\left[\frac{1}{\lambda} \mid \begin{array}{c}
(1-k, 1 / \mu) \\
(n, 1)
\end{array}\right] \quad \text { for } \mu>1
$$

and with the help of identity (A.3) we can write the analytic form of $P_{n}$ in terms of a legal H-function also for $\mu<1$ :

$$
P_{n}=\frac{1}{n ! \Gamma(k)} \mathrm{H}_{1,1}^{1,1}\left[\lambda \mid \begin{array}{c}
(1-n, 1) \\
(k, 1 / \mu)
\end{array}\right] \quad \text { for } 0<\mu<1 .
$$

A reparametrization of Eqs. (11) and (12) using $\langle n\rangle$ in place of the scale parameter $\lambda$ is more convenient. The factorial moments of $P_{n}$ are equivalent to the ordinary moments of $\psi(z)$ for Eq. (1) therefore $\langle n\rangle=\langle z\rangle$ and Eq. (3) yields

$$
\lambda=\frac{\Gamma(k+1 / \mu)}{\Gamma(k)\langle n\rangle} .
$$

For unit scaling exponent $\mu$ the HNBD reduces to the pure NBD given by Eq. (4) which is the $\mu=1$ marginal case of Eq. (11) for $\langle n\rangle<k$ and of Eq. (12) for $\langle n\rangle>k$. This can be deduced by comparing Eq. (10) with (A.9) and recalling that ${ }_{1} \mathrm{~F}_{0}(a,-; x)$ yields the binomial function $(1-x)^{-a}$ for $|x|<1$.

At first glance it may seem strange that a three-parameter discrete probability law requires three different expressions for $P_{n}$ each having a restricted domain of validity over the parameter space. But this complication is a reasonable price for the high degree of flexibility of the HNBD. It involves as a special or limiting case the Poisson transform of many widely used probability densities such as the

- gamma distribution for $\mu=1$ and $k>0$,

- Weibull distribution for $k=1$ and $\mu>0$,

- chi distribution for $\mu=2$ and $k>0$,

- Pareto distribution for $k \rightarrow 0$ and $\mu \rightarrow \infty$,

- log-normal distribution for $\mu \rightarrow 0$ and $k \rightarrow \infty$

to mention but a few. Therefore one may think the HNBD as a family of different discrete probability laws rather than a single distribution. Since the infinite divisibility of $\psi(z)$ is preserved by $P_{n}$ for Eq. (1) we can use Bondesson's theorem [11] to deduce that the HNBD is infinitely divisible if $0 \leq \mu \leq 1$, otherwise this feature of $P_{n}$ is violated.

Before turning our attention to data analysis let us consider briefly the computational aspects of the HNBD. Unfortunately, the Fox function is not available in software packages for the computation of special functions. A straightforward way to calculate $P_{n}$ in fitting procedures is the evaluation of the integral Eq. (1) numerically with $f(x)$ given by Eq. (2), requireing $\langle z\rangle=1$ and changing $z$ to $\langle n\rangle z$ in the Poisson weight. For $\mu \rightarrow 0$ and $k \rightarrow \infty$ the 
HNBD converges to the Poisson transformed log-normal distribution which lacks a representation in terms of known functions. To include this important limiting case into the fits Eq. (2) should be written in the following form [7]:

$$
\psi(z)=\frac{|p|}{\Gamma\left(p^{-2}\right) \sigma z} \exp \left[p^{-2} w-e^{w}\right] \quad \text { for } p \neq 0
$$

where $w=p(\ln z+\alpha) / \sigma$ with new parameters $\sigma>0, p$ and $\alpha$. This reparametrization of the generalized gamma density allows $\mu<0$ as well $(\mu=p / \sigma)$ and the log-normal distribution can be mapped to the origin in $p$,

$$
\psi(z)=\frac{1}{\sqrt{2 \pi} \sigma z} \exp \left[-\frac{(\ln z-\alpha)^{2}}{2 \sigma^{2}}\right] \quad \text { for } p=0 .
$$

Restricting the location parameter $\alpha$ to

$$
\alpha= \begin{cases}\ln \Gamma\left(p^{-2}+\sigma / p\right)-\ln \Gamma\left(p^{-2}\right) & \text { for } p \neq 0 \\ -\sigma^{2} / 2 & \text { for } p=0\end{cases}
$$

$P_{n}$ is computed by inserting Eqs. (14) and (15) into Eq. (1), changing $z$ to $\langle n\rangle z$ in the Poisson weight and evaluating the integral by the Romberg method or Gaussian quadrature [12] or using the numerical integration package of Mathematica [13].

Applying the HNBD to experimental data we shall consider typical reactions and energies where the pure NBD is claimed to be in contradiction with observations. Significant deviations from $\mu=1$ are expected in

1. inelastic $p p$ reactions up to ISR energies,

2. deep-inelastic $e^{+} p$ scattering at HERA energies,

3. $e^{+} e^{-}$annihilations at LEP energies,

4. non-diffractive $p \bar{p}$ collisions at top SPS energy $\sqrt{s}=900 \mathrm{GeV}$.

Items 2 and 3 have already been investigated in refs. $[7,8]$ and here only a few additions will be made. But let us first consider inelastic $p p$ reactions, perhaps the earliest typical example for the failure of NBD fits. Without the aim of completeness we have analysed multiplicity distributions in the c.m. energy range $\sqrt{s}=10.7-546 \mathrm{GeV}$ [14] including the inelastic $p \bar{p}$ data of the UA5 Collaboration. In the fitting procedure numerical evaluation of the integral Eq. (1) was carried out as described above. After some initial playing with the parameters it was found that the $k=1$ special case of the HNBD, i.e. the Poisson transformed Weibull distribution, provides good description of the investigated data. The results of fits are collected in Table 1. It is worth noticing that the best-fit value of $\mu$ slowly decreases with increasing energy, further, $\mu>1$ for each data set. According to some preliminary results the $k=1$ special case produces similar quality fits for inelastic $\pi^{ \pm} p$ and $K^{ \pm} p$ reactions.

The Poisson transformed Weibull law works successfully in deep-inelastic $e^{+} p$ scattering at HERA energies as well. In ref. [8] the multiplicity distributions measured by the H1 Collaboration [15] were compared to the Weibull case of the HNBD neglecting systematic errors of $P_{n}$. To avoid possible misunderstanding we repeated the analysis with the inclusion of both sources of experimental uncertainties. The average multiplicity $\langle n\rangle$ was fixed at its observed value and only $\mu$ was treated as free parameter in the fitting procedure. The results are collected in Table 2, the quoted errors of $\mu$ are statistical and systematic. The success of the $k=1$ special case of the HNBD (even with omitted systematic errors of $P_{n}$ ) suggests that the inelastic $p p$ and deep-inelastic $e^{+} p$ multiplicity distributions are similarly shaped. This seems to be confirmed by the observation [15] that the H1 data and the best-fit NBD deviate from each other at small multiplicities. Due to the diffractive fraction of events, the same happens for inelastic $p p$ data too. In both reactions the rise of $P_{n}$ at small $n$ is less rapid than that of the NBD and this property is well described by the Poisson transform of the Weibull distribution with $\mu>1$.

A completely different type of deviation from $\mu=1$ arises in non-diffractive $p \bar{p}$ collisions at $\sqrt{s}=900 \mathrm{GeV}$. The multiplicity distributions measured by the UA5 Collaboration [16] are at present the highest energy published data for $P_{n}$. The Negative Binomial fits are satisfactory in narrow pseudorapidity intervals but for $|\eta|>2.5$ the NBD fails to reproduce the observed shape of $P_{n}$. According to widespread opinion the failure of the NBD is caused by the much quoted shoulder structure. In narrow bins this is not visible but for $|\eta|>2.5$ some kind of structure can indeed be seen in the tail of $P_{n}$. However, the dominant source of discrepancy between the NBD and observations has nothing to do with the shoulder. It was demonstrated already in [16] that the real difficulty for wide $\eta$-intervals is the pronounced narrow peak of the heavy tailed distributions. Performing HNBD fits reveals clearly that the $\mu=1$ special case is unable to reproduce a highly skewed shape which is so characteristic of the UA5 data for $|\eta|>2.5$. But 
letting $\mu$ to vary freely in the fits it turns out that the $\mu \rightarrow 0, k \rightarrow \infty$ limiting case of the HNBD, i.e. the Poisson transformed log-normal distribution, yields reasonable description of $P_{n}$ possessing a narrow peak and extended tail. The results for full phase-space and wide $\eta$-windows are collected in Table 3. Although systematic deviations between the experimental and theoretical $P_{n}$ inevitably occur due to the shoulder structure (the HNBD is unimodal) the overall shape of the multiplicity distributions can be reproduced remarkably well by the two-parameter Poisson transformed log-normal law. For the full phase-space data the fit is illustrated in Fig. 1.

Interestingly, the log-normal limit of the HNBD was found to be successful also in $e^{+} e^{-}$annihilations at the $Z^{0}$ peak. In ref. [7] the results of three-parameter HNBD fits were presented favouring $\mu \approx 0$ for the full phase-space multiplicity distributions. Here we provide the outcome of two-parameter Poisson transformed log-normal fits, see Table 4. The analysed data sets now include the Aleph data with updated systematic errors and the Opal data corresponding to $\sqrt{s}=161 \mathrm{GeV}$ [17]. As is seen the quality of fits are satisfactory for each experiment. Similar results can be obtained for the Aleph data measured in central rapidity windows [18], these are quoted in Table 5. We have to mention that the Delphi data in $y$-intervals exhibit significant shoulder structure which can not be reproduced by unimodal distributions and therefore the HNBD fails as well.

The importance of the log-normal distribution to approximate $P_{n}$ in $e^{+} e^{-}$and $p \bar{p}$ collisions has already been stressed in the literature [19]. One may think that the observed success of the Poisson transformed log-normal law is only another manifestation of the above cited results. But the widely known and accepted evidences for the lognormality of $P_{n}$ at present energies are not rarely questionable. Their comparison to our findings (which indicate that log-normality emerges asymptotically) will be treated in a separate paper [20].

In conclusion, we have investigated the H-function extension of the NBD obtained by the Poisson transform of the generalized gamma distribution Eq. (2). The analytic form of $P_{n}$ was rederived via Mellin transform. Since Eq. (2) involves as special and limiting cases many classical probability densities, the HNBD is expected to obey high degree of flexibility. Our applications confirmed this expectation. Fitting the HNBD to various multiplicity data claimed to be in disagreement with the pure NBD we have obtained satisfactory results. According to these findings, two major types of departure from $\mu=1$ arise in multiparticle production. For inelastic $p p$ and deep-inelastic $e^{+} p$ scattering the dominant source of discrepancy is the less steep rise of $P_{n}$ at small multiplicities caused by the diffractive component of the underlying dynamics (at least for $p p$ data). The multiplicity distributions can be reproduced successfully by the Weibull case of the HNBD with $\mu>1$. The other $\mu \neq 1$ type behaviour arises in non-diffractive $p \bar{p}$ collisions at $\sqrt{s}=900 \mathrm{GeV}$ where the NBD is incompatible with the highly skewed shape of $P_{n}$ for $|\eta|>2.5$. These heavy tailed distributions with a narrow peak are best described if $\mu \rightarrow 0$ and $k \rightarrow \infty$, i.e. by the log-normal limit of the HNBD. Despite of the absence of skew multiplicity curves, the same type of departure from the pure NBD has been observed also in $e^{+} e^{-}$annihilations at LEP energies. This remarkable ubiquity of the $\mu \rightarrow 0$ limit for the $e^{+} e^{-}$and $p \bar{p}$ data at top energies has a natural explanation based on renormalization group arguments for asymptotic KNO scaling [20]. It will be interesting to see how precisely can the Poisson transformed log-normal law reproduce the forthcoming $p \bar{p}$ data at $\sqrt{s}=1800 \mathrm{GeV}$ at Tevatron.

The observed success of the HNBD indicates that the majority of existing multiplicity data can be interpreted on the basis of the asymptotic KNO scaling form of the Negative Binomial Distribution with the extension of its validity to positive powers of the scaling variable. It is hoped that in the next 25 years the HNBD will provide a helpful unifying framework to study the NB regularity and the origin of possible deviations.

\section{ACKNOWLEDGEMENTS}

I am indebted to T. Csörgö for the helpful comments on the manuscript and to G. Jancsó for the constructive criticisms. This work was supported by the Hungarian Science Foundation under Grant No. OTKA-T024094/1997. 


\section{Appendix: The H-function of Fox}

The Fox function $\mathrm{H}_{\mathrm{p}, \mathrm{q}}^{\mathrm{m}, \mathrm{n}}(x)$ is defined by the Mellin-Barnes type integral

$$
\mathbf{H}_{\mathbf{p}, \mathbf{q}}^{\mathbf{m}, \mathbf{n}}\left[x \mid \begin{array}{c}
\left(a_{p}, \alpha_{p}\right) \\
\left(b_{q}, \beta_{q}\right)
\end{array}\right]=\mathrm{H}_{\mathbf{p}, \mathbf{q}}^{\mathbf{m}, \mathbf{q}}\left[x \mid \begin{array}{c}
\left(a_{1}, \alpha_{1}\right), \ldots,\left(a_{p}, \alpha_{p}\right) \\
\left(b_{1}, \beta_{1}\right), \ldots,\left(b_{q}, \beta_{q}\right)
\end{array}\right]=\frac{1}{2 \pi i} \int_{\mathcal{C}} \frac{A(s) B(s)}{C(s) D(s)} x^{s} d s
$$

with $x \neq 0$ and

$$
\begin{array}{ll}
A(s)=\prod_{j=1}^{m} \Gamma\left(b_{j}-\beta_{j} s\right), & B(s)=\prod_{j=1}^{n} \Gamma\left(1-a_{j}+\alpha_{j} s\right), \\
C(s)=\prod_{j=m+1}^{q} \Gamma\left(1-b_{j}+\beta_{j} s\right), & D(s)=\prod_{j=n+1}^{p} \Gamma\left(a_{j}-\alpha_{j} s\right) .
\end{array}
$$

Above $m, n, p, q$ are integers satisfying $0 \leq n \leq p$ and $1 \leq m \leq q$. In the cases $n=0, q=m$ and $p=n$ (A.2) has to be interpreted as $B(s)=1, C(s)=1$ and $D(s)=1$, respectively. The parameters $\left(a_{1}, \ldots, a_{p}\right)$ and $\left(b_{1}, \ldots, b_{q}\right)$ are complex, whereas parameters $\left(\alpha_{1}, \ldots, \alpha_{p}\right)$ and $\left(\beta_{1}, \ldots, \beta_{q}\right)$ are positive numbers. They are restricted by the condition that $\alpha_{j}\left(b_{h}+\nu\right) \neq \beta_{h}\left(a_{j}-1-\lambda\right)$ for $\nu, \lambda=0,1, \ldots ; h=1, \ldots, m ; j=1, \ldots, n$. The contour $\mathcal{C}$ in the complex $s$ plane is such that the points $s=\left(b_{h}+\nu\right) / \beta_{h}$ and $s=\left(a_{j}-1-\nu\right) / \alpha_{j}$ lie to the right and left of $\mathcal{C}$ respectively while $\mathcal{C}$ extends from $s=\infty-i k$ to $s=\infty+i k$ where $k$ is a constant with $k>\left|\operatorname{Im} b_{h}\right| / \beta_{h}$.

The Fox function makes sense only if the following two existence conditions are satisfied:

1. $x \neq 0$ and $\kappa>0$ with $\kappa=\sum_{j=1}^{q} \beta_{j}-\sum_{j=1}^{p} \alpha_{j}$

2. $\kappa=0$ and $0<|x|<1 / \rho$ with $\rho=\prod_{j=1}^{p} \alpha_{j}^{\alpha_{j}} \prod_{j=1}^{q} \beta_{j}^{-\beta_{j}}$.

Under these conditions $\mathrm{H}(x)$ is an analytic function for $x \neq 0$, in general multivalued, one-valued on the Riemann surface of $\ln x$.

Elementary properties of the Fox function very useful to manipulate them are

$$
\begin{aligned}
& \mathrm{H}_{\mathrm{p}, \mathrm{q}}^{\mathrm{m}, \mathrm{n}}\left[\begin{array}{l|c}
\frac{1}{x} & \left(a_{p}, \alpha_{p}\right) \\
\left(b_{q}, \beta_{q}\right)
\end{array}\right]=\mathrm{H}_{\mathbf{q}, \mathrm{p}}^{\mathrm{n}, \mathrm{m}}\left[x \mid \begin{array}{c}
\left(1-b_{q}, \beta_{q}\right) \\
\left(1-a_{p}, \alpha_{p}\right)
\end{array}\right] \\
& x^{c} \mathrm{H}_{\mathrm{p}, \mathrm{q}}^{\mathrm{m}, \mathrm{n}}\left[x \mid \begin{array}{c}
\left(a_{p}, \alpha_{p}\right) \\
\left(b_{q}, \beta_{q}\right)
\end{array}\right]=\mathrm{H}_{\mathrm{p}, \mathrm{q}}^{\mathrm{m}, \mathrm{n}}\left[x \mid \begin{array}{c}
\left(a_{p}+c \alpha_{p}, \alpha_{p}\right) \\
\left(b_{q}+c \beta_{q}, \beta_{q}\right)
\end{array}\right] \\
& \mathrm{H}_{\mathbf{p}, \mathbf{q}}^{\mathrm{m}, \mathrm{q}}\left[\begin{array}{l|l}
x^{c} & \left(a_{p}, \alpha_{p}\right) \\
\left(b_{q}, \beta_{q}\right)
\end{array}\right]=\frac{1}{c} \mathrm{H}_{\mathrm{p}, \mathbf{q}}^{\mathrm{m}, \mathrm{q}}\left[\begin{array}{l|l}
x & \left(a_{p}, \alpha_{p} / c\right) \\
\left(b_{q}, \beta_{q} / c\right)
\end{array}\right] \quad c>0 .
\end{aligned}
$$

Many of the integral transforms of $\mathrm{H}(x)$ yield Fox functions again with altered parameters. For example, the Laplace transform is given by the equation pair

$$
\mathcal{L}\left\{\mathrm{H}_{\mathbf{p}, \mathbf{q}}^{\mathrm{m}, \mathrm{n}}(c x), r\right\}=\frac{1}{c} \mathrm{H}_{\mathbf{q}, \mathbf{p}+1}^{\mathrm{n}+1, \mathbf{m}}\left[\frac{r}{c} \mid \begin{array}{c}
\left(1-b_{q}-\beta_{q}, \beta_{q}\right) \\
(0,1),\left(1-a_{p}-\alpha_{p}, \alpha_{p}\right)
\end{array}\right] \quad \text { for } 0 \leq \kappa \leq 1
$$

and

$$
\mathcal{L}\left\{\mathrm{H}_{\mathbf{p}, \mathbf{q}}^{\mathrm{m}, \mathrm{n}}(c x), r\right\}=\frac{1}{c} \mathrm{H}_{\mathrm{p}+1, \mathbf{q}}^{\mathrm{m}, \mathrm{n}+1}\left[\begin{array}{c|c}
\frac{c}{r} & (1,1),\left(a_{p}+\alpha_{p}, \alpha_{p}\right) \\
\left(b_{q}+\beta_{q}, \beta_{q}\right)
\end{array}\right] \quad \text { for } \kappa \geq 1
$$

with scale parameter $c>0$. The Mellin transform of $\mathrm{H}(x)$ reads

$$
\mathcal{M}\left\{\mathrm{H}_{\mathbf{p}, \mathbf{q}}^{\mathrm{m}, \mathrm{n}}(c x), s\right\}=\frac{\prod_{j=1}^{m} \Gamma\left(b_{j}+\beta_{j} s\right) \prod_{j=1}^{n} \Gamma\left(1-a_{j}-\alpha_{j} s\right)}{\prod_{j=m+1}^{q} \Gamma\left(1-b_{j}-\beta_{j} s\right) \prod_{j=n+1}^{p} \Gamma\left(a_{j}+\alpha_{j} s\right)} c^{-s} .
$$

An important special case of $\mathrm{H}(x)$ is the generalized hypergeometric function ${ }_{\mathrm{p}} \mathrm{F}_{\mathrm{q}}(x)$. Its relation to the Fox function is given by

$$
\begin{aligned}
& \frac{\prod_{j=1}^{p} \Gamma\left(a_{j}\right)}{\prod_{j=1}^{q} \Gamma\left(b_{j}\right)}{ }_{\mathrm{p}} \mathrm{F}_{\mathbf{q}}\left[\begin{array}{c}
a_{1}, \ldots, a_{p} \\
b_{1}, \ldots, b_{q}
\end{array} \mid x\right]=\mathrm{H}_{\mathbf{p}, \mathbf{q}+1}^{1, \mathfrak{p}}\left[-x \mid \begin{array}{c}
\left(1-a_{p}, 1\right) \\
(0,1),\left(1-b_{q}, 1\right)
\end{array}\right] \\
& =\mathrm{H}_{\mathrm{q}+1, \mathrm{p}}^{\mathrm{p}, 1}\left[-\frac{1}{x} \mid \begin{array}{c}
(1,1),\left(b_{q}, 1\right) \\
\left(a_{p}, 1\right)
\end{array}\right] \quad \text { for } p \leq q \text {, or } p=q+1 \text { and }|x|<1 \text {. }
\end{aligned}
$$

For more details on the H-function as well as for a rich collection of its particular cases the reader is referred to [9]. 
[1] Z. Koba, H.B. Nielsen and P. Olesen, Nucl. Phys. B40, 314 (1972).

[2] A. Giovannini, Nuovo Cim. 10A, 713 (1972).

[3] P. Carruthers and C.C. Shih, Int. J. Mod. Phys. A2, 1447 (1987).

[4] I.M. Dremin, Physics Uspekhi 37, 715 (1994).

[5] R. Ugoccioni, A. Giovannini and S. Lupia, in Proc. XXIV Int. Symp. on Multiparticle Dynamics, p. 384, eds. A. Giovannini, S. Lupia and R. Ugoccioni (World Scientific, 1995).

[6] N.L. Johnson and S. Kotz, Distributions in Statistics Vol. 2., Continuous Univariate Distributions (Wiley, 1970).

[7] S. Hegyi, Phys. Lett. B387, 642 (1996).

[8] S. Hegyi, Phys. Lett. B388, 837 (1996).

[9] A.M. Mathai and R.K. Saxena, The H-Function with Applications in Statistics and Other Disciplines (Wiley Eastern, 1978).

[10] F. Oberhettinger, Tables of Mellin Transforms (Springer, 1974).

[11] L. Bondesson, Scand. Actuar. J. 48 (1978).

[12] W.H. Press, B.P. Flannery, S.A. Teukolsky and W.T. Vetterling, Numerical Recipes - The Art of Scientific Computing (Cambridge Univ. Press, 1986).

[13] S. Wolfram, Mathematica - A System for Doing Mathematics by Computer (Addison-Wesley, 1991).

[14] C. Bromberg et al., Phys. Rev. D15, 64 (1977).

J. Erwin et al., Phys. Rev. Lett. 32, 254 (1974).

C. Bromberg et al., Phys. Rev. Lett. 31, 1563 (1973).

D. Brick et al., Phys. Rev. D25, 2794 (1982).

A.E. Brenner et al., Phys. Rev. D26, 1497 (1982).

S. Barish et al., Phys. Rev. D9, 2689 (1974).

M. Adamus et al., Z. Phys. C32, 475 (1986).

F.T. Dao et al., Phys. Rev. Lett. 29, 1627 (1972).

J.L. Bailly et al., Z. Phys. C23, 205 (1984).

R. Ammar et al., Phys. Lett. B178, 124 (1986).

A. Breakstone et al., Phys. Rev. D30, 528 (1984).

G.J. Alner et al., Phys. Rep. 154, 247 (1987).

[15] S. Aid et al., Z. Phys. C72, 573 (1996).

[16] R.E. Ansorge et al., Z. Phys. C43, 357 (1989).

[17] R. Barate et al., CERN-PPE/96-186, to appear in Phys. Rep.

P. Abreau et al., Z. Phys. C50, 185 (1991).

B. Adeva et al., Z. Phys. C55, 39 (1992).

P.D. Acton et al., Z. Phys. C53, 539 (1992).

K. Ackerstaff et al., CERN-PPE/97-015, submitted to Z. Phys. C.

[18] D. Buskulic et al., Z. Phys. C69, 15 (1995).

[19] R. Szwed, G. Wrochna and A.K. Wróblewski, Mod. Phys. Lett. A5, 1851 (1990).

S. Carius and G. Ingelman, Phys. Lett. B252, 647 (1990).

[20] S. Hegyi, manuscript in preparation. 


\begin{tabular}{cccc}
\hline \hline$\sqrt{s}(\mathrm{GeV})$ & $\mu$ & $\langle n\rangle$ & $\chi^{2} /$ d.o.f. \\
\hline 10.7 & $5.353 \pm 1.431$ & $5.503 \pm 0.171$ & $1.7 / 6$ \\
13.8 & $3.064 \pm 0.298$ & $6.465 \pm 0.151$ & $4.5 / 8$ \\
13.9 & $3.248 \pm 0.250$ & $6.258 \pm 0.112$ & $4.5 / 8$ \\
16.6 & $2.765 \pm 0.087$ & $6.955 \pm 0.071$ & $16.3 / 11$ \\
18.2 & $2.892 \pm 0.551$ & $7.407 \pm 0.410$ & $2.1 / 8$ \\
19.7 & $2.942 \pm 0.160$ & $7.603 \pm 0.128$ & $7.8 / 11$ \\
21.7 & $2.575 \pm 0.125$ & $7.838 \pm 0.129$ & $15.4 / 12$ \\
23.9 & $2.678 \pm 0.225$ & $8.735 \pm 0.224$ & $13.5 / 11$ \\
26.0 & $2.725 \pm 0.113$ & $9.077 \pm 0.114$ & $8.5 / 11$ \\
27.6 & $2.485 \pm 0.101$ & $8.869 \pm 0.136$ & $16.7 / 14$ \\
30.4 & $2.468 \pm 0.150$ & $9.341 \pm 0.261$ & $5.6 / 15$ \\
38.8 & $2.503 \pm 0.076$ & $10.045 \pm 0.112$ & $11.9 / 14$ \\
44.0 & $2.349 \pm 0.135$ & $10.703 \pm 0.223$ & $4.5 / 17$ \\
52.6 & $2.329 \pm 0.109$ & $11.457 \pm 0.212$ & $10.4 / 19$ \\
62.2 & $2.392 \pm 0.105$ & $12.267 \pm 0.258$ & $17.5 / 18$ \\
546.0 & $1.840 \pm 0.034$ & $27.255 \pm 0.292$ & $50.9 / 45$ \\
\hline \hline
\end{tabular}

TABLE I. Results of HNBD fits (Weibull case, $k=1$ ) to the multiplicity distributions measured in inelastic $p p$ reactions [14]. The last row corresponds to the inelastic $p \bar{p}$ data of the UA5 Collaboration.

\begin{tabular}{lrcc}
\hline \hline$\eta^{*}$-interval & $W(\mathrm{GeV})$ & $\mu$ & $\chi^{2} /$ d.o.f. \\
\hline $1<\eta^{*}<2$ & $80 \div 115$ & $1.916 \pm 0.194$ & $1.6 / 13$ \\
& $115 \div 150$ & $1.872 \pm 0.085$ & $0.6 / 14$ \\
& $150 \div 185$ & $1.891 \pm 0.212$ & $1.5 / 14$ \\
& $185 \div 220$ & $1.841 \pm 0.203$ & $0.8 / 14$ \\
\hline $1<\eta^{*}<3$ & $80 \div 115$ & $2.336 \pm 0.166$ & $2.5 / 17$ \\
& $115 \div 150$ & $2.116 \pm 0.143$ & $2.4 / 18$ \\
& $150 \div 185$ & $2.041 \pm 0.143$ & $0.9 / 20$ \\
& $185 \div 220$ & $2.094 \pm 0.155$ & $0.6 / 21$ \\
\hline $1<\eta^{*}<4$ & $80 \div 115$ & $3.488 \pm 0.385$ & $0.8 / 18$ \\
& $115 \div 150$ & $3.100 \pm 0.180$ & $2.2 / 20$ \\
& $150 \div 185$ & $2.920 \pm 0.226$ & $1.5 / 22$ \\
& $185 \div 220$ & $2.733 \pm 0.192$ & $1.6 / 22$ \\
\hline $1<\eta^{*}<5$ & $80 \div 115$ & $4.811 \pm 0.392$ & $0.9 / 18$ \\
& $115 \div 150$ & $4.238 \pm 0.277$ & $2.1 / 21$ \\
& $150 \div 185$ & $4.011 \pm 0.288$ & $1.9 / 22$ \\
& $185 \div 220$ & $4.021 \pm 0.334$ & $1.5 / 23$ \\
\hline \hline
\end{tabular}

TABLE II. Results of HNBD fits (Weibull case, $k=1$ ) to the deep-inelastic $e^{+} p$ multiplicity data of the H1 Collaboration [15]. The average multiplicity $\langle n\rangle$ was fixed at its experimental value. 


\begin{tabular}{lccc}
\hline \hline$\eta$-interval & $\sigma$ & $\langle n\rangle$ & $\chi^{2} /$ d.o.f. \\
\hline$\Delta \eta=3.0$ & $0.702 \pm 0.020$ & $22.050 \pm 0.546$ & $30.2 / 81$ \\
$\Delta \eta=5.0$ & $0.621 \pm 0.018$ & $32.747 \pm 0.780$ & $35.9 / 99$ \\
full phase-space & $0.538 \pm 0.014$ & $35.552 \pm 0.834$ & $32.7 / 52$ \\
\hline \hline
\end{tabular}

TABLE III. Results of HNBD fits (log-normal case, $p=0$ ) to the UA5 non-diffractive $p \bar{p}$ multiplicity data for $|\eta|>2.5$ at $\sqrt{s}=900 \mathrm{GeV}[16]$. The $(p, \sigma)$ parametrization of the HNBD is discussed in the text.

\begin{tabular}{lccr}
\hline \hline Experiment & $\sigma$ & $\langle n\rangle$ & $\chi^{2} /$ d.o.f. \\
\hline Aleph & $0.202 \pm 0.008$ & $21.094 \pm 0.226$ & $11.2 / 25$ \\
Delphi & $0.201 \pm 0.004$ & $21.353 \pm 0.104$ & $32.4 / 24$ \\
L3 & $0.210 \pm 0.009$ & $20.742 \pm 0.254$ & $15.9 / 21$ \\
Opal & $0.213 \pm 0.005$ & $21.349 \pm 0.103$ & $17.2 / 25$ \\
Opal, $161 \mathrm{GeV}$ & $0.235 \pm 0.016$ & $24.417 \pm 0.423$ & $3.9 / 23$ \\
\hline \hline
\end{tabular}

TABLE IV. Results of HNBD fits (log-normal case, $p=0$ ) to the $e^{+} e^{-}$full phase-space multiplicity distributions at $\sqrt{s}=91 \mathrm{GeV}$ (top four rows) and $161 \mathrm{GeV}[17]$.

\begin{tabular}{lcrr}
\hline \hline$y$-interval & $\sigma$ & $\langle n\rangle$ & $\chi^{2} /$ d.o.f. \\
\hline$\Delta y=0.5$ & $0.565 \pm 0.046$ & $3.058 \pm 0.143$ & $3.5 / 18$ \\
$\Delta y=1.0$ & $0.547 \pm 0.030$ & $6.426 \pm 0.231$ & $9.3 / 32$ \\
$\Delta y=1.5$ & $0.505 \pm 0.018$ & $9.897 \pm 0.219$ & $26.3 / 38$ \\
$\Delta y=2.0$ & $0.454 \pm 0.021$ & $13.284 \pm 0.293$ & $13.7 / 44$ \\
\hline \hline
\end{tabular}

TABLE V. Results of HNBD fits (log-normal case, $p=0$ ) to the Aleph multiplicity data in central rapidity windows [18].

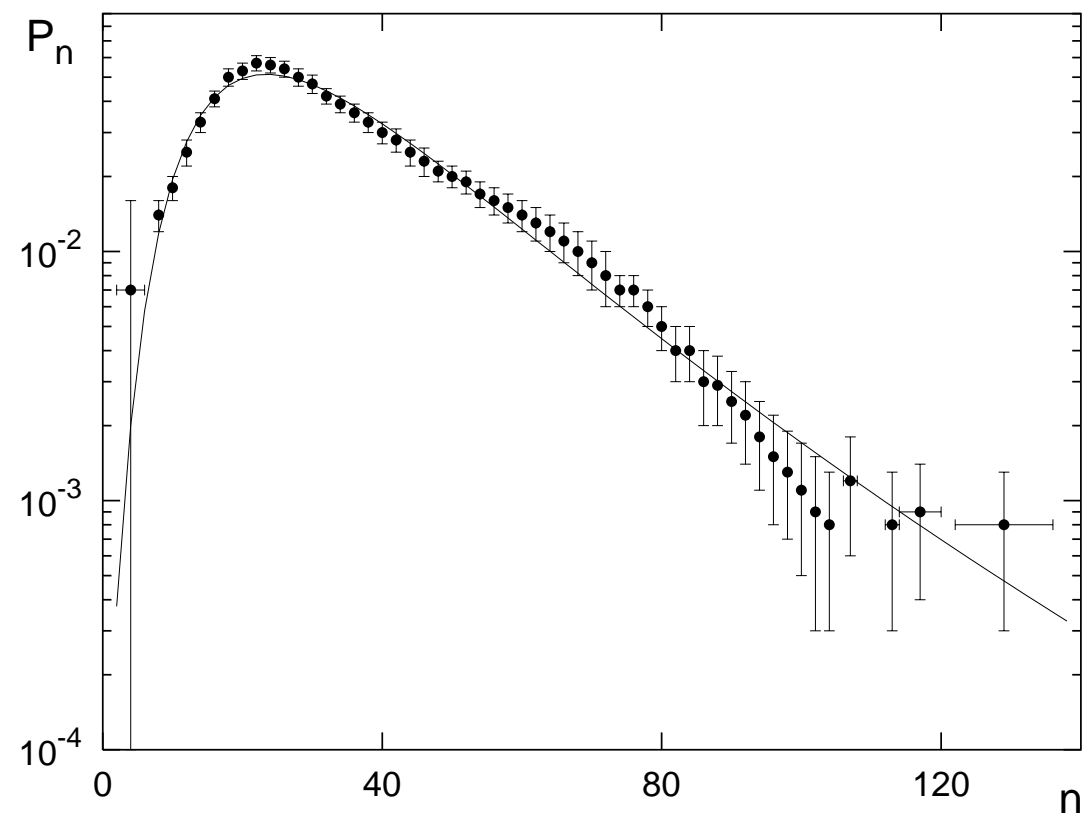

FIG. 1. HNBD fit (lognormal case, $p=0$ ) to the non-diffractive $p \bar{p}$ multiplicity distribution in full phase-space at $\sqrt{s}=900 \mathrm{GeV}$. 